\title{
"Crossover" children in the youth justice and child protection systems
}

\author{
Susan Baidawi and Rosemary Sheehan \\ Routledge, UK and New York, 2020 \\ ISBN: 978-0-367-26110-8, pp.146, hardback, NZD 110.01
}

$\mathrm{H}$

aving one's prejudices confirmed (Ahaa - so I was right about that!), is always a pleasant feeling-a mixture of smug self-righteousness and a sense of, perhaps, being the teeniest bit clever.

If this book was preaching to my particular choir, there is still a strong rationale for its thesis-a disproportionate number of young people progress from the Child Protection system ("victim") to the Youth Justice system ("offender").

So it was as I read my way through a fairly dense 146 pages of academic critique of Youth Justice jurisdictions across a number of countries, including the United States, United Kingdom, Canada, New Zealand and Australia.

This book examines the outcomes for children and young people who are involved in both child protection and youth justice systems: "crossover" children.

Susan Baidawi is Senior Research Fellow and Rosemary Sheehan is Professor at the Department of Social Work, Monash University, Australia. Their book has been meticulously researched over two years using an original study of 300 Australian children/young people aged 10 to 17 years, "investigating the nexus between child maltreatment and adversity, child welfare and youth offending" (p. 31).

The study combined two data collection methods: a detailed audit of Children's Court case files and key stakeholder consultation and focus groups. This is based on an Ecological Systems framework where micro (family, peers, school, etc.), meso (neighbourhood, work, etc.) and macro (cultural values, beliefs, laws, etc.) systems are considered. This data collection method also gives anecdotal information statistical credibility.

There is an established relationship between child maltreatment and youth offending and this research seeks to understand how the respective systems operate and collaborate, especially in cases where children/young people are concurrently or historically involved with both systems.

In my late teens, armed with a strong sense of social justice and little else, I could see that people's judgements appeared to fall into two distinct camps. Take, for example, the aetiology of racism. Are some races inherently lazy / criminally minded/ aggressive/intellectually deficient etc., or is there something pathological in our socioeconomic systems which creates unfair disadvantages for people who differ from the racial or cultural norm?

Although my argument was not particularly nuanced at the time, I could still see that people appeared to subscribe to either the "blame-the-individual/ race-based" pathology or the "blame-systemic racism" pathology.

I was in the latter camp and as I trained and worked as a social worker, everything I learnt and witnessed merely reinforced this view. Whilst working in Care \& Protection 
(and long before reading this elucidating book), I saw how children with unresolved trauma and loss moved from the Child Protection system to the Youth Justice one. They may as well have had the letters and an arrow tattooed on their foreheads, so predictable seemed their trajectory.

This book comprises nine chapters, each exploring a different aspect of this issueincluding the introduction of the topic and its identification as an international trend, through to crossover children's characteristics and pathways, the role of culture and indigeneity, the nature and contexts of offending, where criminogenic risk meets systemic disadvantage and finally "disrupting the [care to custody] pipeline" (p. 124).

Given that the care-to-custody pipeline has been internationally recognised, this book seeks to answer the question as to what experiences distinguish the lives of those children with child protection backgrounds who also have contact with the youth justice system.

The reverse does not appear to be trueless than $3 \%$ of those involved with child protection services will have justice system involvement as juveniles (p. 1).

Interestingly, poor parental supervision and parental rejection (i.e., emotional neglect and abuse), are the most consistent predictors of youth offending. Coming from a social work background in care and protection (with a strong focus on attachment), this did not surprise me.

In the form of a disclaimer, nothing in this book actually surprised me but this is not to take away from the thorough research and important conclusions.

You could read Chapter 1, "Child protection and youth offending", along with the conclusions of the other chapters and have pretty much the whole book nailed. This is the strength of the book, not a failing. Its ongoing reinforcement of the major theme that crossover children are disadvantaged from birth (sometimes even in utero) through individual, familial, social, economic and legal systems is impossible to ignore or disagree with.

The dilemma can be summed up in one phrase from an Alternative Education professional: crossover children "had a rough go from day dot." (p. 66) Furthermore, systemic risks compound the risks of each other-they are not merely additive, but cumulative. Indigenous populations experience the double whammy of systemic deprivation compounded by colonisation and consequent institutional racism.

The statistics are compelling: "childhood maltreatment nearly doubles the risk of later violent offending throughout adolescence and adulthood" (p. 11).

As a forensic social worker in the Youth Courts, I see the correlation between childhood adversity and youth justice involvement on a daily basis. Children leave neglectful homes that are full of conflict and find other young people who come from similar backgrounds-an important validating experience in its own right. School attendance can become sporadic with no parent to oversee this process. The further behind a young person slips in their education, the less relevant school becomes. Disadvantage piles on disadvantage and the path of crime is almost inevitable-how else will you eat? The pull of gangs as an alternative whānau is indeed strong-we all need to be understood by someone.

Within the dynamic of crossover kids are two competing philosophies: should the youth justice system be therapeutic (given the crossover children's care and protection backgrounds) or punitive? What would therapeutic jurisprudence even look like, if professionals could agree on its necessity?

Chapter 9, "Disrupting the pipeline: decriminalising child protection-involved 
youth" sums up the best approach. At last, I was thinking, having been rather overwhelmed by the negative statistics which brutally document young people's distressing lives.

No real surprises here, either, but good to have my beliefs validated. What is needed is a "whole-of-government approach" (p. 124), including the education, health, welfare, housing, income support and disability sectors. Early intervention (yep, that old chestnut), intensive family support, traumainformed responsivity and specialised, crossover children's courts to co-ordinate overall responses.

If this review reads more as a summary of the book than a critique, I am unapologetic. This is an important book-the evidence is out there and we would be wise to take heed.

Reviewed by Fritha Parkes, Registered social worker, Auckland, New Zealand 